\title{
Ethylene Signaling Facilitates Plant Adaption to Physical Barriers
}

\author{
Simu Liu' ${ }^{*}$ and Hui Chen ${ }^{2 *}$ \\ ' Guangdong Provincial Key Laboratory for Plant Epigenetics, College of Life Sciences and Oceanography, Shenzhen \\ University, Shenzhen, China, ${ }^{2}$ Guangdong Key Laboratory of Genome Instability and Human Disease, School of Medicine, \\ Shenzhen University, Shenzhen, China
}

\section{OPEN ACCESS}

Edited by:

Anna N. Stepanova,

North Carolina State University,

United States

Reviewed by:

Keith Lindsey,

Durham University, United Kingdom

Irina Ivanova Vaseva,

Bulgarian Academy of Sciences

(BAS), Bulgaria

Satoko Yoshida,

Nara Institute of Science and Technology (NAIST), Japan

Brad M. Binder,

The University of Tennessee,

Knoxville, United States

*Correspondence:

Simu Liu

liusm@szu.edu.cn

Hui Chen

huichen@szu.edu.cn

huichen_0408@163.com

Specialty section:

This article was submitted to

Plant Physiology,

a section of the journal

Frontiers in Plant Science

Received: 20 April 2021

Accepted: 02 July 2021

Published: 29 July 2021

Citation:

Liu S and Chen H (2021) Ethylene Signaling Facilitates Plant Adaption

to Physical Barriers.

Front. Plant Sci. 12:697988.

doi: 10.3389/fp/s.2021.697988
The morphological changes are usually observed in the terrestrial plants to respond to physical barriers. The phytohormone ethylene plays an essential role in the morphological development of plants encountering exogenous mechanical impedance, which enables plants to grow optimally in response to physical barriers. Ethylene is shown to regulate these developmental processes directly or in concert with other phytohormones, especially auxin. In this mini review, the involvement of ethylene action in seedling emergence from the soil, root movement within the soil, and parasitic plant invasion of the host plant are described.

Keywords: ethylene signaling, mechanical impedance, morphological changes, seedling emergence, root elongation, parasitic plant invasion

\section{INTRODUCTION}

In natural environments, the life cycle of terrestrial plants usually starts with the germination of seeds buried under the soil. After germination, seedlings need to successfully fulfill emergence from the soil to establish a photoautotrophic lifestyle, and the roots of seedling should forage soil for mineral nutrients and water acquisition. During these processes, the shoots and roots are subjected to mechanical stress from the soil. In addition, parasitic plants, whose survival depends on the host plants, encounter the physical barrier provided by the host plants, thereby they need to penetrate through the host tissues to access nutrients. To acclimate to these physical barriers, plants adopt a developmental strategy by which they adjust their morphological modifications in accordance with the mechanical impedance (Leivar et al., 2008; Yoshida et al., 2016; Gommers and Monte, 2018; Vaseva et al., 2018), such as hypocotyl thickening, apical hook formation, and root shortening occur within autotrophic plants and haustoria emergence within parasitic plants.

It has been reported that mechanical impedance boosts the production of ethylene (Goeschl et al., 1966; Zhong et al., 2014). As a gaseous plant hormone, ethylene diffuses rapidly and regulates diverse developmental and physiological processes in plants such as seedling emergence, root elongation, leaf senescence, fruit ripening, root nodulation, as well as biotic and abiotic stress responses (Bleecker and Kende, 2000). It is not surprising that terrestrial plants employ ethylene as the vital signal molecule to regulate the directional growth of plant organs since the ethylene 
signaling system existed prior to the plant colonization of land (Ju et al., 2015). In this mini review, we aimed to summarize current advances regarding ethylene regulation of morphological changes in facilitating autotrophic plant adaption to soilprovided physical barriers, as well as parasitic plant conquest to host plant-created physical barriers.

\section{ETHYLENE SIGNALING PATHWAY}

In 1901, Dimitry Neljubov described the effects of ethylene on the growth of etiolated pea seedlings, a morphology that by 1913 was termed as the "triple response" in some dicots showing shorter and thicker hypocotyl, an exaggerated apical hook, and a shorter and swelling root (Bakshi et al., 2015). This response was utilized to genetically screen the mutants with altered ethylene response in dicotyledonous Arabidopsis thaliana (Bleecker et al., 1988; Guzmán and Ecker, 1990). The main components of ethylene signaling have been identified, including five endoplasmic reticulum (ER)-localized receptors, namely, ethylene response 1 (ETR1), ethylene response sensor 1 (ERS1), ETR2, ERS2, and ethylene insensitive 4 (EIN4), the negative regulator, namely, constitutive triple response 1 (CTR1), the central mediator EIN2, and the master transcription factors, namely, ethylene insensitive 3 (EIN3) and EIN3-like 1 (EIL1).

The ethylene signaling pathway in plants is well characterized (Figure 1A). In the absence of ethylene, the active receptors recruit CTR1 to phosphorylate EIN2 and thus lead to EIN2 degradation mediated by the $26 \mathrm{~S}$ proteasome, which represses the downstream signaling pathway. In the presence of ethylene, the receptors are inactivated, reducing CTR1-mediated EIN2 phosphorylation and consequently resulting in EIN2 C-terminal end (EIN2-C) cleavage from ER-bound EIN2-N portion (Ju et al., 2012; Qiao et al., 2012). The EIN2-C enters the cytoplasmic P-body, where it represses the translation of EIN3-degrading factors, namely, EIN3-binding F-box proteins 1 and 2 (EBF1/2) and stabilizes EIN3/EIL1 proteins (Li et al., 2015; Merchante et al., 2015). EIN2-C is also able to translocate from the cytoplasm to the nucleus, where it associates with a histone binding protein for epigenetically facilitating EIN3/EIL1-dependent transcriptional regulation (Zhang et al., 2017). EIN3/EIL1 can function as a transcriptional activator or repressor in response to ethylene (Wang et al., 2020).

Although the linear ethylene signaling pathway is conserved between monocotyledonous rice and dicotyledonous Arabidopsis (Yang et al., 2015a), the exogenous application of ethylene inhibits root growth but promotes coleoptile growth of etiolated rice seedlings (Ma et al., 2013). Different from Arabidopsis EIN3 and EIL1, which regulate both shoot and root responses to ethylene, the rice EIN3 transcription factors, namely, OsEIL1 and OsEIL2, mediate the inhibition of root elongation and promotion of coleoptile elongation, respectively (Yang et al., 2015b). Unlike rice, ethylene inhibits coleoptile elongation in other monocots, including maize, wheat, sorghum, and Brachypodium distachyon (Yang et al., 2015a). Considering that rice is cultivated mainly in the frequently flooded river deltas, ethylene-promoted tissue elongation via gibberellin may contribute to diminish flooding stress (Hattori et al., 2009). These findings suggest a pivotal role of ethylene in modulating plant developmental processes to acclimate to ever-changing environments.

\section{ETHYLENE SIGNALING IS REQUIRED FOR SHOOTS TO EMERGE FROM THE SOIL}

To overcome the soil barrier and reach sunlight, seedlings frequently adopt a developmental strategy dynamically adjusting their morphological structures in accordance to soil depth (Zhong et al., 2014). In Arabidopsis, the short and thick hypocotyl is suitable for enhancing the lifting capacity of etiolated seedlings, and the apical hook is an elegant structure for protecting the delicate shoot apical meristem against the mechanical damage when seedlings emerge from the soil. In rice, the coleoptile and mesocotyl are the two essential structures of seedlings responsible for moving toward the soil surface (Lee et al., 2017; Xiong et al., 2017). The coleoptile, which wraps the emergent shoot, safeguards the plumule against mechanical injures. The mesocotyl, a structure between the coleoptile node and the basal part of the seminal root in etiolated monocot seedlings, pushes the buds out of the soil. It has been reported that the soil-imposed mechanical impedance stimulates the production of ethylene (Goeschl et al., 1966; Zhong et al., 2014; Xiong et al., 2017), which acts as a key signal linking plant responses to the environmental cues (Sarquis et al., 1991; He et al., 1996).

\section{ETHYLENE SUPPRESSES HYPOCOTYL ELONGATION IN RESPONSE TO SOIL BARRIER}

In Arabidopsis, the subterranean hypocotyl growth during soil emergence is an important phase for seedling establishment and survival. Under the soil, ethylene employs diverse pathways to suppress the hypocotyl elongation and increase the hypocotyl strength (Figure 1B), hence improves the capacity of hypocotyl response to soil barrier. Ethylene activates the ethylene response factor 1 (ERF1) pathway in an EIN3-dependent manner to slow down hypocotyl elongation and thus enables seedlings to penetrate through the soil layers more easily (Zhong et al., 2014). Ethylene is also able to inhibit hypocotyl cell elongation by upregulating wave-dampened2-like 5 (WDL5) gene expression through the EIN3-dependent signaling pathway (Sun et al., 2015). WDL5 encodes a microtubule-associated protein that stabilizes the microtubule of cortical cells in the etiolated hypocotyl. In addition, ethylene suppresses pectin degradation and thus enhances cell wall stiffness by EIN3-mediated downregulation of polygalacturonase involved in expansion 3 (PGX3) gene expression (Wu et al., 2020). This EIN3-PGX3 regulatory module contributes to the inhibition of hypocotyl elongation as well as the facilitation of seedling soil emergence. Although ethylene inhibition of hypocotyl elongation may delay seedlings reaching the sunlight, a longer period of etiolated growth is 
B

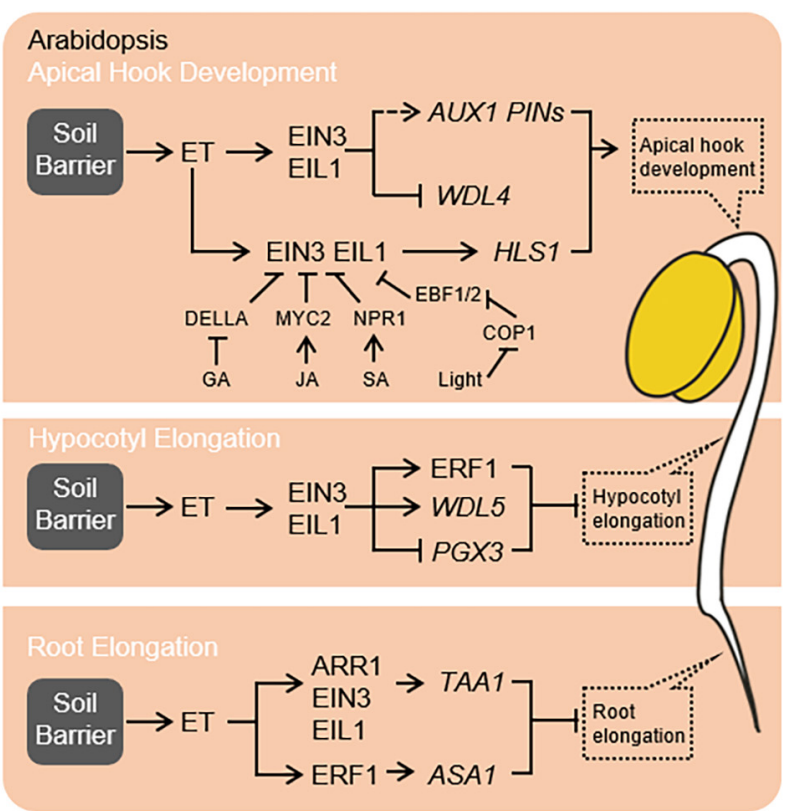

A

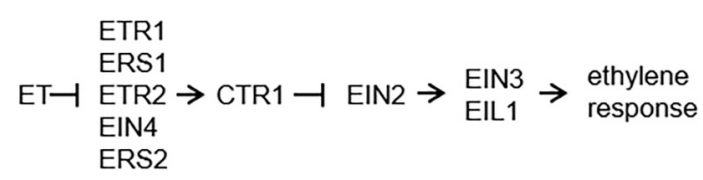

C

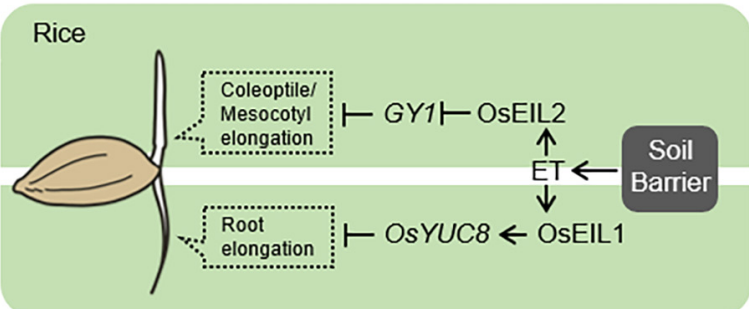

D

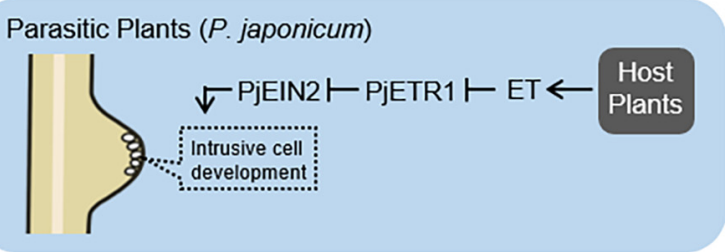

FIGURE 1 | Ethylene signaling regulates the growth and development of plants encountering physical barriers. (A) The linear model of the ethylene signaling pathway in Arabidopsis. Ethylene signaling involves ethylene receptors [ethylene response 1 (ETR1), ethylene response sensor 1 (ERS1), ETR2, ethylene insensitive 4 (EIN4), and ERS2], the negative regulator [constitutive triple response 1 (CTR1)], the central mediator (EIN2), and the master transcription factors [EIN3/EIN3-like 1 (EIL1)]. The linear ethylene signaling pathway is conserved between rice and Arabidopsis (Yang et al., 2015a). (B) Ethylene facilitates Arabidopsis adaption to soil barriers. Ethylene regulates apical hook development by two different pathways. On the one hand, ethylene reinforces asymmetric auxin distribution within the apical hypocotyl by inducing the expression of AUX1 and PINs, and by repressing the expression of WDL4 that influences PIN protein trafficking (Vandenbussche et al., 2010; Zádníková et al., 2010; Deng et al., 2021). On the other hand, ethylene regulates differential growth of apical hypocotyl through EIN3/EIL1 transcriptional regulation of HLS1 expression (Shen et al., 2016). Other endogenous phytohormones including gibberellic acid, jasmonic acid, and salicylic acid and light signal also modulate ethylene-regulated apical hook development by influencing the EIN3/EIL1-HLS1 module (An et al., 2012; Zhang et al., 2014; Shi et al., 2016a; Huang et al., 2020). Ethylene inhibits hypocotyl elongation by activation of ERF1 pathway, upregulation of WDL5 expression, and downregulation of PGX3 expression via EIN3-dependent signaling pathway (Zhong et al., 2014; Sun et al., 2015; Wu et al., 2020). Ethylene inhibits root elongation by induction of auxin biosynthesis through upregulating WE/8/TAA1 and ASA1 expression in EIN3- and ARR1-dependent and ERF1-dependent manner, respectively (Stepanova et al., 2005, 2008; Mao et al., 2016; Yan et al., 2017). (C) Ethylene facilitates rice adaption to soil barriers. In monocot rice seedlings, ethylene promotes both coleoptile and mesocotyl elongation by repression of GY1 expression via OsEIL2-dependent signaling pathway (Xiong et al., 2017). Ethylene inhibits root growth by promoting auxin biosynthesis through inducing OsYUC8 expression in an OsEIL1-dependent manner (Qin et al., 2017). (D) Ethylene signaling is required for the parasitic plant to invade the host plant. In the parasitic plant Phtheirospermum japonicum, the PjETR1 and PjEIN2, homologs of Arabidopsis ETR1 and EIN2, are required for intrusive cell development in response to ethylene produced by the host plants (Cui et al., 2020).

beneficial for EIN3-phytochrome interacting factor 3 (PIF3)modulated preassembly of photosynthetic machinery, which is necessary for the seedling transition from dark to light (Zhong et al., 2012, 2014).

\section{ETHYLENE REGULATES APICAL HOOK DEVELOPMENT DURING SOIL EMERGENCE}

The differential distribution of auxin across the apical hypocotyl region and accumulation on the concave (inner) is essential for regulating asymmetric cell elongation between concave and convex (outer) and consequently leading to apical hook formation (Raz and Ecker, 1999; Béziat and Kleine-Vehn, 2018). Auxin distribution is facilitated by the auxin influx carrier auxinresistant 1 (AUX1) and the efflux carrier pin-formed (PIN) family proteins (Petrásek and Friml, 2009). During the early phases of seedling germination, a gravity-stimulated and PIN2directed asymmetric auxin distribution in the root is able to extend into hypocotyl and thus initiates the establishment of asymmetric auxin distribution in this zone (Zhu et al., 2019). Ethylene-induced expression of AUX1 and PINs within the apical hypocotyl may further reinforce this initial auxin asymmetry (Vandenbussche et al., 2010; Zádníková et al., 2010). Moreover, ethylene represses the expression of WDL4 via the EIN3dependent signaling pathway, which influences PIN protein trafficking and auxin asymmetric distribution (Figure 1B; Deng et al., 2021). Then, the transmembrane kinase 1 (TMK1)mediated auxin response pathway translates differential cellular auxin to differential cell growth during apical hook formation (Cao et al., 2019). A recent finding also supports that PINfacilitated polar auxin transport followed by TMK-mediated auxin signaling pathway is required for mechanically induced apical hook formation (Baral et al., 2021).

Besides depending on the auxin pathway, ethylene regulates apical hook development through EIN3/EIL1 transcriptional 
regulation of hookless 1 (HLS1) expression (EIN3/EIL1-HLS module) (Figure 1B; Shen et al., 2016). HLS1 encodes a putative $\mathrm{N}$-acetyltransferase, and the loss of function of the HLS1 gene completely disrupts apical hook curvature (Lehman et al., 1996), indicating an essential role of HLS1 in apical hook formation. Although the biochemical nature of HLS1 protein remains mysterious, the formation of HLS1 oligomer is required for its function during apical hook formation (Lyu et al., 2019). Other endogenous phytohormones and light signal also modulate apical hook development by influencing the EIN3/EIL1-HLS1 module. In darkness, the gibberellinrepressed DELLA, jasmonic acid-activated MYC2, or salicylic acid-activated NPR1 can directly interact with and alter the transcriptional activity of EIN3/EIL1 (An et al., 2012; Zhang et al., 2014; Huang et al., 2020). The photomorphogenic central repressor constitutive photomorphogensis 1 (COP1) is also able to stabilize EIN3/EIL1 protein by directly ubiquitinating EBF1/2 for degradation (Shi et al., 2016a). It is still unknown how HLS1 regulates asymmetric cell growth during apical hook formation. A potential explanation may be that HLS1 mediates ethylene-repressed accumulation of auxin response factor 2 (ARF2) protein, which is an auxin response transcription factor negatively regulating differential auxin response during apical hook formation (Li et al., 2004).

Upon seedling reaching the soil surface, the mechanical forcestimulated production of ethylene decreases, whereas the light exposure increases. The light represses COP1 nuclear localization and thus releases EBF1/2 for EIN3 degradation (Shi et al., 2016a). Meanwhile, the light activates the photoreceptor phytochrome $\mathrm{B}$ (PhyB) and promotes its translocation into the nucleus, where it enhances EBF1/2-stimulated EIN3/EIL1 degradation and disrupts HLS1 oligomer formation as well (Shi et al., 2016b; Lyu et al., 2019). Therefore, the light-regulated EIN3/EIL1 reduction inactivates ethylene signaling, which attenuates hook curvature but facilitates hook opening and cotyledon expansion to initiate photomorphogenic growth programs.

\section{ETHYLENE PROMOTES ELONGATION OF COLEOPTILE AND MESOCOTYL IN RICE}

In Arabidopsis seedlings, thickened hypocotyl and apical hook are the key morphological structures facilitating the soil emergence, while in rice seedlings they are coleoptile and mesocotyl. Ethylene-promoted coleoptile and mesocotyl elongation are mediated by transcription factor OsEIL2 in rice seedlings (Figure 1C). OsEIL2 directly represses transcription of the gaoyao 1 (GY1), which encodes a phospholipase that functions at the initial step of jasmonic acid biosynthesis (Xiong et al., 2017). Consistent with this, ethylene emission increases while jasmonic acid content decreases in the rice seedlings with increasing seed-sowing depth of soil, suggesting that ethylene stimulates coleoptile and mesocotyl elongation to facilitate seedling emergence by inhibition of jasmonic acid biosynthesis (Xiong et al., 2017). Overexpression of OsEIN2 also leads to a longer coleoptile and longer mesocotyl of etiolated rice seedlings in the presence of ethylene ( $\mathrm{Ma}$ et al., 2013), indicating a major role of ethylene in promoting coleoptile and mesocotyl elongation in rice seedlings. Other phytohormones such as brassinosteroids and strigolactones are also involved in regulating mesocotyl growth (Sun et al., 2018; Zheng et al., 2020). How ethylene coordinately with other hormones modulates mesocotyl elongation during rice seedling emergence from the soil needs more detailed studies.

\section{ETHYLENE SIGNALING IS REQUIRED FOR ROOT ADAPTION TO SOIL BARRIER}

In most cases, the shoots encounter soil barriers only before they emerge from the soil, whereas the roots have to face persistent soil barriers in the whole life cycle. Thus, the roots need to continuously adjust their morphological structures to respond to ever-changing local soil environments. The physically impeded roots display diverse morphological alterations, such as inhibition of root elongation and increase of radial dimension, resembling the morphological changes observed when roots are grown in the presence of ethylene (Jacobsen et al., 2021). Consistent with these observations, when ethylene biosynthesis or signaling is repressed, the degree of root response to mechanical impedance is significantly alleviated (Santisree et al., 2011). Interestingly, the loss of function of WDL5 gene, which is a target of EIN3, impairs mechanical stress-inhibited elongation of both hypocotyl and root (Sun et al., 2015; Okamoto et al., 2021), suggesting that a general mechanism regulating cell elongation should be crucial for ethylene regulation of morphological changes in response to mechanical impedance.

The root cap, which is located at the apex of the root and protects the root apical meristem, is able to sense mechanical stimulus and control the direction of root growth (Dreyer and Edelmann, 2018). Removal of root cap disturbs ethylene-inhibited root growth and affects root exploration and penetration in the soils, indicating that the root cap should be a potential site of the ethylene-regulated root-soil interaction (Hahn et al., 2008). When in contact with the soil, the root cap needs to evaluate the resistance strength of the soil and then takes the decision to penetrate through the soil layers or avoidance of obstacles. Charles and Francis Darwin have described this intelligent root cap in their book "The Power of Movement of Plants" (Darwin and Darwin, 1881). Ethylene emission and accumulation around the root tip tissues may be crucial for root caps to choose the above two different strategies according to the soil conditions. Ethylene-promoted increased diameter of roots may lead to favorable mechanical strength to penetrate through the penetrable soil layers; alternatively, ethylene-inhibited root elongation may facilitate avoidance of compacted soils. In agreement with this view, compacted soil-restricted ethylene diffusion triggers a rapid and sustained increase of EIN3-green fluorescent protein in cell nuclei at the root elongation zone, indicating that ethylene signaling is strongly activated in this zone. Consistently, the roots of Osein 2 and Oseil1 mutants 
that are insensitive to ethylene penetrate compacted soil more effectively than wild-type's roots (Pandey et al., 2021).

In screening for mutants that display ethylene insensitivity in roots of Arabidopsis, mutants related to auxin biosynthesis, auxin transport, and auxin signaling were identified (Stepanova et al., 2007). In Arabidopsis, the main auxin is indole-3-acetic acid (IAA), which is mainly synthesized by the indole-3-pyruvic acid (IPA) pathway using tryptophan (Trp) as a precursor (Zhao, 2012). Upon perception of auxin, transport inhibitor response 1 (TIR1) and auxin signaling F-box proteins (AFBs) physically interact with auxin or IAA (Aux/IAA) transcriptional repressors and promote the degradation of them, which release ARF transcription factors to activate diverse auxin-responsive genes (Chapman and Estelle, 2009).

The weak ethylene insensitive 2/anthranilate synthase alpha 1 (WEI2/ASA1) and WEI7/anthranilate synthase beta 1 (ASB1) genes encode rate-limiting enzyme of Trp biosynthesis. Ethylene inhibits root elongation by upregulation of WEI2/ASA1 and WEI7/ASB1 to lead to auxin accumulation in the root tip, and ERF1 is shown to physically bind to the ASA1 promoter and induces its expression (Stepanova et al., 2005; Mao et al., 2016). The WEI8/tryptophan aminotransferase of Arabidopsis 1 (TAA1) and Yucca (YUC) encode two key enzymes in the IPA pathway and catalyze the conversion of Trp to IPA and IPA to IAA, respectively (Zhao, 2012). EIN3 regulates WEI8/TAA1 expression by interacting with and enhancing response regulator 1 (ARR1) transcriptional activity (Figure 1B; Stepanova et al., 2008; Yan et al., 2017). In rice, OsEIL1 is able to directly target the OsYUC8 promoter to regulate its expression (Figure 1C; Qin et al., 2017). In addition, reduced expression of rice auxin receptor genes OsTIR1 and OsAFB2 cause root ethylene insensitive (Chen et al., 2018). Furthermore, loss of function of soil-surface rooting 1 (SOR1), an E3 ubiquitin ligase modulating protein stability of OsIAA26 and OsIAA9, also leads to defective of root ethylene response (Chen et al., 2018). A recent finding shows that auxin is involved in root-obstacle avoidance and especially the PINmediated polar auxin transport facilitates this process (Lee et al., 2020). These findings indicate that ethylene largely employs the auxin pathway to regulate root growth.

\section{ETHYLENE SIGNALING IS REQUIRED FOR THE PARASITIC PLANT TO INVADE THE HOST PLANT}

Unlike most of plants whose shoots and roots mainly encounter soil-caused physical barriers, parasitic plants majorly experience another type of physical barrier organized by the host plants (Twyford, 2018). The parasitic plants need to obtain all or part of nutrients from host plants to support their survival, so they form a special morphological structure termed haustoria to attach to hosts, penetrate through the host tissues, and ultimately establish the physiological conduit (Clarke et al., 2019). The Orobanchaceae parasitic plants are root parasitic plants that establish a connection with host plants by the formation of haustorium. The Triphysaria versicolor and Phtheirospermum japonicum belong to Orobanchaceae have been studied in the laboratory to expand our understanding of communication between parasitic and host plants.

After germination, Orobanchaceae parasitic plants initiate haustorium formation since they recognize active haustoriuminducing factors (HIFs) such as 2,6-dimethoxy- $p$-benzoquinone (DMBQ), produced by the potential hosts. Upon contact to host roots, a haustorium initiates invasion using the intrusive cells potentially differentiated from epidermal cells at the apex. In $T$. versicolor, the production of ethylene increases in response to $\mathrm{DMBQ}$, and the blockage of ethylene biosynthesis or signaling almost completely suppresses haustorium formation, suggesting that ethylene is involved in haustorium development (Tomilov et al., 2005). To uncover the genetic programs of haustorium development, a genetic screening was performed using P. japonicum, and the mutation of PjETR1 and PjEIN2, encoding a homolog of Arabidopsis ETR1 and EIN2, respectively, were identified (Figure 1D). Both mutants displayed elongated haustorium but failed host invasion. Further observations suggest that parasitic plants initiate haustorium development when they detect HIFs and maintain haustorium elongation until they sense host-derived ethylene, which inhibits haustorium elongation but promotes differentiation of haustorium apex cells into intrusive cells for host invasion (Cui et al., 2020). These findings indicate that ethylene signaling is essential for parasitic plants to overcome physical barriers provided by host plants.

\section{CONCLUSION AND PERSPECTIVES}

As a plant hormone, ethylene promotes the environmental adaption of plants by regulating diverse morphological development. In this mini review, we described the role of ethylene in facilitating plant adaption to diverse physical barriers for survival. To establish a photoautotrophic lifestyle, ethylene promotes hypocotyl shortening and apical hook formation in Arabidopsis and both coleoptile and mesocotyl elongation in rice to support them to move through the soils more effectively and safely. To uptake water and mineral nutrients from the soils, ethylene modulates root growth for optimal response to soil barriers. To establish a successful parasitic relationship, ethylene promotes host invasion by stimulating intrusive cell development at the haustorium apex.

Although some targets regulated directly by EIN3/EIL1 have been identified (Figure 1), it remains largely unclear how mechanical impedance triggers ethylene action. Mechanosensitive ion channels are a common mechanism for sensing mechanical impedance. In Arabidopsis, the MID1complementing activity 1 (MCA1) is a $\mathrm{Ca}^{2+}$-permeable mechanosensitive channel enabling roots to overcome mechanical barriers (Okamoto et al., 2021), and the piezo1 (PZO1) is another ion channel that affects $\mathrm{Ca}^{2+}$ transients in response to mechanical stimulation and is required for roots to penetrate hard agar (Mousavi et al., 2021). The ethylene is biosynthesized by two dedicated enzymatic reactions. The substrate $S$-adenosyl-L-methionine is converted to the ethylene precursor 1-aminocyclopropane-1-carboxylic acid (ACC) by the ACC synthase (ACS), and then the ACC is converted to ethylene 
by the ACC oxidase (ACO) (Pattyn et al., 2021). In Vigna radiata, the AIM-1 is a key ACS, whose mRNA levels dramatically increase in the presence of mechanical stimulation (Botella et al., 1995). In Arabidopsis, the ACS6 expression was induced by touch (Arteca and Arteca, 1999). It will be interesting to study whether and how mechano-sensitive ion channel-mediated increase of cytoplasmic $\mathrm{Ca}^{2+}$ upregulates ACS gene expression and thus triggers ethylene production and signaling.

Characterizing the role of ethylene in facilitating plant adaption to physical barriers at the levels of molecular biology, biochemistry, and cellular biology is therefore important not only to expand our understanding of the plant-soil/plantplant interaction but also to breed crops that can grow optimally in unfavorable lands with compact soils and develop a useful strategy to control parasitic plant-caused cereal yield losses.

\section{REFERENCES}

An, F., Zhang, X., Zhu, Z., Ji, Y., He, W., Jiang, Z., et al. (2012). Coordinated regulation of apical hook development by gibberellins and ethylene in etiolated Arabidopsis seedlings. Cell Res. 22, 915-927. doi: 10.1038/cr.2012.29

Arteca, J. M., and Arteca, R. N. (1999). A multi-responsive gene encoding 1 -aminocyclopropane-1-carboxylate synthase (ACS6) in mature Arabidopsis leaves. Plant Mol. Biol. 39, 209-219.

Bakshi, A., Schemansky, J. M., Chang, C., and Binder, B. M. (2015). History of research on the plant hormone ethylene. J. Plant Growth Regul. 34, 809-827. doi: 10.1007/s00344-015-9522-9

Baral, A., Aryal, B., Jonsson, K., Morris, E., Demes, E., Takatani, S., et al. (2021). External mechanical cues reveal a katanin-independent mechanism behind auxin-mediated tissue bending in plants. Dev. Cell 56, 67-80.e63.

Béziat, C., and Kleine-Vehn, J. (2018). The road to auxin-dependent growth repression and promotion in apical hooks. Curr. Biol. 28, R519-R525.

Bleecker, A. B., and Kende, H. (2000). Ethylene: a gaseous signal molecule in plants. Annu. Rev. Cell Dev. Biol. 16, 1-18. doi: 10.1146/annurev.cellbio.16.1.1

Bleecker, A. B., Estelle, M. A., Somerville, C., and Kende, H. (1988). Insensitivity to ethylene conferred by a dominant mutation in Arabidopsis thaliana. Science 241, 1086-1089. doi: 10.1126/science.241.4869.1086

Botella, J. R., Arteca, R. N., and Frangos, J. A. (1995). A mechanical strain-induced 1-aminocyclopropane-1-carboxylic acid synthase gene. Proc. Natl. Acad. Sci. U S A 92, 1595-1598. doi: 10.1073/pnas.92.5.1595

Cao, M., Chen, R., Li, P., Yu, Y., Zheng, R., Ge, D., et al. (2019). TMK1-mediated auxin signalling regulates differential growth of the apical hook. Nature 568, 240-243. doi: 10.1038/s41586-019-1069-7

Chapman, E. J., and Estelle, M. (2009). Mechanism of auxin-regulated gene expression in plants. Annu. Rev. Genet. 43, 265-285. doi: 10.1146/annurevgenet-102108-134148

Chen, H., Ma, B., Zhou, Y., He, S. J., Tang, S. Y., Lu, X., et al. (2018). E3 ubiquitin ligase SOR1 regulates ethylene response in rice root by modulating stability of Aux/IAA protein. Proc. Natl. Acad. Sci. U S A 115, 4513-4518. doi: 10.1073/ pnas. 1719387115

Clarke, C. R., Timko, M. P., Yoder, J. I., Axtell, M. J., and Westwood, J. H. (2019). Molecular Dialog Between Parasitic Plants and Their Hosts. Annu. Rev. Phytopathol. 57, 279-299. doi: 10.1146/annurev-phyto-082718-100043

Cui, S., Kubota, T., Nishiyama, T., Ishida, J. K., Shigenobu, S., Shibata, T. F., et al. (2020). Ethylene signaling mediates host invasion by parasitic plants. Sci. Adv. 6:eabc2385. doi: 10.1126/sciadv.abc2385

Darwin, C., and Darwin, F. (1881). The Power of Movement in Plants. New York, NY: D. Appleton\&Co.

Deng, J., Wang, X., Liu, Z., and Mao, T. (2021). The microtubule-associated protein WDL4 modulates auxin distribution to promote apical hook opening in Arabidopsis. Plant Cell doi: 10.1093/plcell/koab080 [Online ahead of print].

\section{AUTHOR CONTRIBUTIONS}

Both authors listed have made a substantial, direct and intellectual contribution to the work, and approved it for publication.

\section{FUNDING}

This study was supported by a grant from the National Natural Science Foundation of China (31800213).

\section{ACKNOWLEDGMENTS}

We apologize to those whose work could not be cited due to space limitations.

Dreyer, J., and Edelmann, H. G. (2018). Root cap-mediated evaluation of soil resistance towards graviresponding roots of maize (Zea mays L.) and the relevance of ethylene. Ann. Bot. 122, 791-800.

Goeschl, J. D., Rappaport, L., and Pratt, H. K. (1966). Ethylene as a factor regulating the growth of pea epicotyls subjected to physical stress. Plant Physiol. 41, 877-884. doi: 10.1104/pp.41.5.877

Gommers, C. M. M., and Monte, E. (2018). Seedling establishment: a dimmer switch-regulated process between dark and light signaling. Plant Physiol. 176, 1061-1074. doi: 10.1104/pp.17.01460

Guzmán, P., and Ecker, J. R. (1990). Exploiting the triple response of Arabidopsis to identify ethylene-related mutants. Plant Cell 2, 513-523. doi: 10.2307/3869113

Hahn, A., Zimmermann, R., Wanke, D., Harter, K., and Edelmann, H. G. (2008). The root cap determines ethylene-dependent growth and development in maize roots. Mol. Plant 1, 359-367. doi: 10.1093/mp/ssm027

Hattori, Y., Nagai, K., Furukawa, S., Song, X. J., Kawano, R., Sakakibara, H., et al. (2009). The ethylene response factors SNORKEL1 and SNORKEL2 allow rice to adapt to deep water. Nature 460, 1026-1030. doi: 10.1038/nature08258

He, C. J., Morgan, P. W., and Drew, M. C. (1996). Transduction of an ethylene signal is required for cell death and lysis in the root cortex of maize during Aerenchyma formation induced by Hypoxia. Plant Physiol. 112, 463-472. doi: 10.1104/pp.112.2.463

Huang, P., Dong, Z., Guo, P., Zhang, X., Qiu, Y., Li, B., et al. (2020). Salicylic acid suppresses apical hook formation via NPR1-mediated repression of EIN3 and EIL1 in Arabidopsis. Plant Cell 32, 612-629. doi: 10.1105/tpc.19.00658

Jacobsen, A. G. R., Jervis, G., Xu, J., Topping, J. F., and Lindsey, K. (2021). Root growth responses to mechanical impedance are regulated by a network of ROS, ethylene and auxin signalling in Arabidopsis. New Phytol. 231, 225-242. doi: $10.1111 / \mathrm{nph} .17180$

Ju, C., Van de Poel, B., Cooper, E. D., Thierer, J. H., Gibbons, T. R., Delwiche, C. F., et al. (2015). Conservation of ethylene as a plant hormone over 450 million years of evolution. Nat. Plants 1:14004.

Ju, C., Yoon, G. M., Shemansky, J. M., Lin, D. Y., Ying, Z. I., Chang, J., et al. (2012). CTR1 phosphorylates the central regulator EIN2 to control ethylene hormone signaling from the ER membrane to the nucleus in Arabidopsis. Proc. Natl. Acad. Sci. US A 109, 19486-19491. doi: 10.1073/pnas.1214848109

Lee, H. J., Kim, H. S., Park, J. M., Cho, H. S., and Jeon, J. H. (2020). PINmediated polar auxin transport facilitates root-obstacle avoidance. New Phytol. 225, 1285-1296. doi: 10.1111/nph.16076

Lee, H. S., Sasaki, K., Kang, J. W., Sato, T., Song, W. Y., and Ahn, S. N. (2017). Mesocotyl elongation is essential for seedling emergence under deep-seeding condition in rice. Rice 10:32.

Lehman, A., Black, R., and Ecker, J. R. (1996). HOOKLESS1, an ethylene response gene, is required for differential cell elongation in the Arabidopsis hypocotyl. Cell 85, 183-194. doi: 10.1016/s0092-8674(00)81095-8

Leivar, P., Monte, E., Oka, Y., Liu, T., Carle, C., Castillon, A., et al. (2008). Multiple phytochrome-interacting bHLH transcription factors repress 
premature seedling photomorphogenesis in darkness. Curr. Biol. 18, 18151823. doi: $10.1016 /$ j.cub.2008.10.058

Li, H., Johnson, P., Stepanova, A., Alonso, J. M., and Ecker, J. R. (2004). Convergence of signaling pathways in the control of differential cell growth in Arabidopsis. Dev. Cell 7, 193-204. doi: 10.1016/j.devcel.2004.07.002

Li, W., Ma, M., Feng, Y., Li, H., Wang, Y., Ma, Y., et al. (2015). EIN2-directed translational regulation of ethylene signaling in Arabidopsis. Cell 163, 670-683. doi: 10.1016/j.cell.2015.09.037

Lyu, M., Shi, H., Li, Y., Kuang, K., Yang, Z., Li, J., et al. (2019). Oligomerization and photo-deoligomerization of HOOKLESS1 controls plant differential cell growth. Dev. Cell 51, 78-88.e3.

Ma, B., He, S. J., Duan, K. X., Yin, C. C., Chen, H., Yang, C., et al. (2013). Identification of rice ethylene-response mutants and characterization of MHZ7/OsEIN2 in distinct ethylene response and yield trait regulation. Mol. Plant 6, 1830-1848. doi: 10.1093/mp/sst087

Mao, J. L., Miao, Z. Q., Wang, Z., Yu, L. H., Cai, X. T., and Xiang, C. B. (2016). Arabidopsis ERF1 mediates cross-talk between ethylene and auxin biosynthesis during primary root elongation by regulating ASA1 expression. PLoS Genet. 12:e1005760. doi: 10.1371/journal.pgen.1005760

Merchante, C., Brumos, J., Yun, J., Hu, Q., Spencer, K. R., Enríquez, P., et al. (2015). Gene-specific translation regulation mediated by the hormonesignaling molecule EIN2. Cell 163, 684-697. doi: 10.1016/j.cell.2015.09.036

Mousavi, S. A. R., Dubin, A. E., Zeng, W. Z., Coombs, A. M., Do, K., Ghadiri, D. A., et al. (2021). PIEZO ion channel is required for root mechanotransduction in Arabidopsis thaliana. Proc. Natl. Acad. Sci. U S A 118:e2102188118. doi: 10.1073/pnas.2102188118

Okamoto, T., Takatani, S., Motose, H., Iida, H., and Takahashi, T. (2021). The root growth reduction in response to mechanical stress involves ethylenemediated microtubule reorganization and transmembrane receptor-mediated signal transduction in Arabidopsis. Plant Cell Rep. 40, 575-582. doi: 10.1007/ s00299-020-02653-6

Pandey, B. K., Huang, G., Bhosale, R., Hartman, S., Sturrock, C. J., Jose, L., et al. (2021). Plant roots sense soil compaction through restricted ethylene diffusion. Science 371, 276-280. doi: 10.1126/science.abf3013

Pattyn, J., Vaughan-Hirsch, J., and Van de Poel, B. (2021). The regulation of ethylene biosynthesis: a complex multilevel control circuitry. New Phytol. 229, 770-782. doi: $10.1111 / \mathrm{nph} .16873$

Petrásek, J., and Friml, J. (2009). Auxin transport routes in plant development. Development 136, 2675-2688. doi: 10.1242/dev.030353

Qiao, H., Shen, Z., Huang, S. S., Schmitz, R. J., Urich, M. A., Briggs, S. P., et al. (2012). Processing and subcellular trafficking of ER-tethered EIN2 control response to ethylene gas. Science 338, 390-393. doi: 10.1126/science.122 5974

Qin, H., Zhang, Z., Wang, J., Chen, X., Wei, P., and Huang, R. (2017). The activation of OsEIL1 on YUC8 transcription and auxin biosynthesis is required for ethylene-inhibited root elongation in rice early seedling development. PLoS Genet. 13:e1006955. doi: 10.1371/journal.pgen.1006955

Raz, V., and Ecker, J. R. (1999). Regulation of differential growth in the apical hook of Arabidopsis. Development 126, 3661-3668. doi: 10.1242/dev.126.16.3661

Santisree, P., Nongmaithem, S., Vasuki, H., Sreelakshmi, Y., Ivanchenko, M. G., and Sharma, R. (2011). Tomato root penetration in soil requires a coaction between ethylene and auxin signaling. Plant Physiol. 156, 1424-1438. doi: 10.1104/pp.111.177014

Sarquis, J. I., Jordan, W. R., and Morgan, P. W. (1991). Ethylene evolution from Maize (Zea mays L.) seedling roots and shoots in response to mechanical impedance. Plant Physiol. 96, 1171-1177. doi: 10.1104/pp.96.4.1171

Shen, X., Li, Y., Pan, Y., and Zhong, S. (2016). Activation of HLS1 by mechanical stress via ethylene-stabilized EIN3 is crucial for seedling soil emergence. Front. Plant Sci. 7:1571.

Shi, H., Liu, R., Xue, C., Shen, X., Wei, N., Deng, X. W., et al. (2016a). Seedlings transduce the depth and mechanical pressure of covering soil using COP1 and ethylene to regulate EBF1/EBF2 for soil emergence. Curr. Biol. 26, 139-149. doi: 10.1016/j.cub.2015.11.053

Shi, H., Shen, X., Liu, R., Xue, C., Wei, N., Deng, X. W., et al. (2016b). The red light receptor Phytochrome B directly enhances substrate-E3 ligase interactions to attenuate ethylene responses. Dev. Cell 39, 597-610. doi: 10.1016/j.devcel.2016. 10.020
Stepanova, A. N., Hoyt, J. M., Hamilton, A. A., and Alonso, J. M. (2005). A link between ethylene and auxin uncovered by the characterization of two rootspecific ethylene-insensitive mutants in Arabidopsis. Plant Cell 17, 2230-2242. doi: $10.1105 /$ tpc. 105.033365

Stepanova, A. N., Robertson-Hoyt, J., Yun, J., Benavente, L. M., Xie, D. Y., Dolezal, K., et al. (2008). TAA1-mediated auxin biosynthesis is essential for hormone crosstalk and plant development. Cell 133, 177-191. doi: 10.1016/j.cell.2008.01. 047

Stepanova, A. N., Yun, J., Likhacheva, A. V., and Alonso, J. M. (2007). Multilevel interactions between ethylene and auxin in Arabidopsis roots. Plant Cell 19, 2169-2185. doi: 10.1105/tpc.107.052068

Sun, J., Ma, Q., and Mao, T. (2015). Ethylene regulates the arabidopsis microtubule-associated protein WAVE-DAMPENED2-LIKE5 in Etiolated Hypocotyl elongation. Plant Physiol. 169, 325-337. doi: 10.1104/pp.15.00609

Sun, S., Wang, T., Wang, L., Li, X., Jia, Y., Liu, C., et al. (2018). Natural selection of a GSK3 determines rice mesocotyl domestication by coordinating strigolactone and brassinosteroid signaling. Nat. Commun. 9:2523.

Tomilov, A. A., Tomilova, N. B., Abdallah, I., and Yoder, J. I. (2005). Localized hormone fluxes and early haustorium development in the hemiparasitic plant Triphysaria versicolor. Plant Physiol. 138, 1469-1480. doi: 10.1104/pp.104. 057836

Twyford, A. D. (2018). Parasitic plants. Curr. Biol. 28, R857-R859.

Vandenbussche, F., Petrásek, J., Zádníková, P., Hoyerová, K., Pesek, B., Raz, V., et al. (2010). The auxin influx carriers AUX1 and LAX3 are involved in auxinethylene interactions during apical hook development in Arabidopsis thaliana seedlings. Development 137, 597-606. doi: 10.1242/dev.040790

Vaseva, I. I., Qudeimat, E., Potuschak, T., Du, Y., Genschik, P., Vandenbussche, F., et al. (2018). The plant hormone ethylene restricts Arabidopsis growth via the epidermis. Proc. Natl. Acad. Sci. U S A 115, E4130-E4139.

Wang, L., Ko, E. E., Tran, J., and Qiao, H. (2020). TREE1-EIN3-mediated transcriptional repression inhibits shoot growth in response to ethylene. Proc. Natl. Acad. Sci. U S A 117, 29178-29189. doi: 10.1073/pnas.2018735117

Wu, Q., Li, Y., Lyu, M., Luo, Y., Shi, H., and Zhong, S. (2020). Touch-induced seedling morphological changes are determined by ethylene-regulated pectin degradation. Sci. Adv. 6:eabc9294. doi: 10.1126/sciadv.abc9294

Xiong, Q., Ma, B., Lu, X., Huang, Y. H., He, S. J., Yang, C., et al. (2017). Ethylene-Inhibited jasmonic acid biosynthesis promotes Mesocotyl/Coleoptile elongation of etiolated rice seedlings. Plant Cell 29, 1053-1072. doi: 10.1105/ tpc. 16.00981

Yan, Z., Liu, X., Ljung, K., Li, S., Zhao, W., Yang, F., et al. (2017). Type B response regulators act as central integrators in transcriptional control of the auxin biosynthesis Enzyme TAA1. Plant Physiol. 175, 1438-1454. doi: 10.1104/pp. 17.00878

Yang, C., Lu, X., Ma, B., Chen, S. Y., and Zhang, J. S. (2015a). Ethylene signaling in rice and Arabidopsis: conserved and diverged aspects. Mol. Plant 8, 495-505. doi: 10.1016/j.molp.2015.01.003

Yang, C., Ma, B., He, S. J., Xiong, Q., Duan, K. X., Yin, C. C., et al. (2015b). MAOHUZI6/ETHYLENE INSENSITIVE3-LIKE1 and ETHYLENE INSENSITIVE3-LIKE2 regulate ethylene response of roots and coleoptiles and negatively affect salt tolerance in rice. Plant Physiol. 169, 148-165. doi: 10.1104/ pp. 15.00353

Yoshida, S., Cui, S., Ichihashi, Y., and Shirasu, K. (2016). The haustorium, a specialized invasive organ in parasitic plants. Annu. Rev. Plant Biol. 67, 643667. doi: 10.1146/annurev-arplant-043015-111702

Zádníková, P., Petrásek, J., Marhavy, P., Raz, V., Vandenbussche, F., Ding, Z., et al. (2010). Role of PIN-mediated auxin efflux in apical hook development of Arabidopsis thaliana. Development 137, 607-617. doi: 10.1242/dev.041277

Zhang, F., Wang, L., Qi, B., Zhao, B., Ko, E. E., Riggan, N. D., et al. (2017). EIN2 mediates direct regulation of histone acetylation in the ethylene response. Proc. Natl. Acad. Sci. U S A 114, 10274-10279. doi: 10.1073/pnas.1707937114

Zhang, X., Zhu, Z., An, F., Hao, D., Li, P., Song, J., et al. (2014). Jasmonate-activated MYC2 represses ETHYLENE INSENSITIVE3 activity to antagonize ethylenepromoted apical hook formation in Arabidopsis. Plant Cell 26, 1105-1117. doi: $10.1105 /$ tpc. 113.122002

Zhao, Y. (2012). Auxin biosynthesis: a simple two-step pathway converts tryptophan to indole-3-acetic acid in plants. Mol. Plant 5, 334-338. doi: 10. 1093/mp/ssr104 
Zheng, J., Hong, K., Zeng, L., Wang, L., Kang, S., Qu, M., et al. (2020). Karrikin signaling acts parallel to and additively with strigolactone signaling to regulate rice Mesocotyl Elongation in darkness. Plant Cell 32, 2780-2805. doi: 10.1105/ tpc. 20.00123

Zhong, S., Shi, H., Xue, C., Wang, L., Xi, Y., Li, J., et al. (2012). A molecular framework of light-controlled phytohormone action in Arabidopsis. Curr. Biol. 22, 1530-1535. doi: 10.1016/j.cub.2012. 06.039

Zhong, S., Shi, H., Xue, C., Wei, N., Guo, H., and Deng, X. W. (2014). Ethyleneorchestrated circuitry coordinates a seedling's response to soil cover and etiolated growth. Proc. Natl. Acad. Sci. U S A 111, 3913-3920. doi: 10.1073/ pnas. 1402491111

Zhu, Q., Gallemi, M., Pospisil, J., Zadnikova, P., Strnad, M., and Benkova, E. (2019). Root gravity response module guides differential growth determining both root bending and apical hook formation in Arabidopsis. Development 146:dev175919.
Conflict of Interest: The authors declare that the research was conducted in the absence of any commercial or financial relationships that could be construed as a potential conflict of interest.

Publisher's Note: All claims expressed in this article are solely those of the authors and do not necessarily represent those of their affiliated organizations, or those of the publisher, the editors and the reviewers. Any product that may be evaluated in this article, or claim that may be made by its manufacturer, is not guaranteed or endorsed by the publisher.

Copyright (c) 2021 Liu and Chen. This is an open-access article distributed under the terms of the Creative Commons Attribution License (CC BY). The use, distribution or reproduction in other forums is permitted, provided the original author(s) and the copyright owner(s) are credited and that the original publication in this journal is cited, in accordance with accepted academic practice. No use, distribution or reproduction is permitted which does not comply with these terms. 